\title{
Abnormal expression of the mitotic checkpoint protein BubR1 contributes to the anti-microtubule drug resistance of esophageal squamous cell carcinoma cells
}

\author{
MIN HU ${ }^{1}$, QINGSONG LIU ${ }^{2}$, PEIPEI SONG ${ }^{1}$, XIAOQIN ZHAN ${ }^{1}$, MIN LUO $^{1}$, CHEN LIU $^{1}$, \\ DANDAN YANG $^{1}$, YAN CAI ${ }^{2}$, FAN ZHANG $^{1}$, FENGBING JIANG $^{1}$, YAN ZHANG ${ }^{1}$, MIN TANG ${ }^{1}$, \\ GUOWEI ZUO ${ }^{1}$, LAN ZHOU ${ }^{1}$, JINYONG LUO ${ }^{1}$, QIONG SHI ${ }^{1}$ and YAGUANG WENG ${ }^{1}$ \\ ${ }^{1}$ Key Laboratory of Diagnostic Medicine designated by The Chinese Ministry of Education and School of \\ Diagnostic Medicine, Chongqing Medical University, Chongqing 400016; ${ }^{2}$ Department of Laboratory Medicine, \\ Affiliated Hospital of North Sichuan Medical College, Nanchong, P.R. China
}

Received September 18, 2012; Accepted October 15, 2012

DOI: $10.3892 /$ or.2012.2117

\begin{abstract}
Esophageal cancer is a common malignancy with a high mortality rate. The lack of effective chemotherapy and a means to overcome drug resistance leads to the predictable failure of esophageal cancer treatment. Mitotic checkpoint proteins play a critical role in regulating the cell cycle and proliferation. Abnormal expression of the mitotic checkpoint protein BubR1 has been reported in several types of cancers. In this study, we investigated the role of BubR1 in conferring resistance of esophageal cancer cells to anti-microtubule drugs. Using quantitative real-time PCR analysis on 50 samples of paired esophageal squamous cell cancer (ESC) tissues and adjacent non-cancerous tissues, we found that $72 \%$ (36 of 50) of the analyzed ESC samples exhibited high expression levels of BubR1, which was also confirmed in ESC cell lines. ESC cells with high levels of BubR1 were less sensitive to the anti-microtubule drugs paclitaxel and nocodazole. Recombinant adenovirus-mediated enforced expression of BubR1 in relatively sensitive ESC cell lines resulted in increased resistance to paclitaxel. Conversely, RNAi-mediated knockdown of BubR1 restored ESC cell sensitivity to paclitaxel. Cell cycle analysis indicated that the sub-G1 population increased in the ESC cells with reduced BubR1 levels. Taken together, our results suggest that upregulation of BubR1 expression may be associated with ESC resistance to paclitaxel treatment. Thus, BubR1 may serve as a potential chemosensitizing target to overcome chemoresistance.
\end{abstract}

Correspondence to: Professor Yaguang Weng or Dr Qiong Shi, School of Diagnostic Medicine, Chongqing Medical University, Chongqing 400016, P.R. China

E-mail: yaguangweng@126.com

E-mail: anniesq8718@126.com

Key words: esophageal squamous cell cancer, mitotic checkpoint protein, BubR1, paclitaxel, chemoresistance

\section{Introduction}

The incidence and mortality of esophageal cancer rank high on the global cancer list, particularly in developing countries (1). Esophageal squamous cell carcinoma (ESC) is one of the most common cancers in China. Due to the lack of early symptoms, rapid progression, ineffective chemotherapy and frequent recurrence after surgery, ESC is characterized by high mortality rate with a 5-year survival rate of less than $10 \%$ (2). Although multi-modality therapy and neoadjuvant therapy have been employed, the survival rate of ESC patients has not improved for many years. The molecular events underlying esophageal cancer development remain undefined. Therefore, there is an urgent need to understand the factors associated with prognosis, therapy and drug resistance of ESCs.

Cell cycle progression is tightly regulated by checkpoint systems which play an essential role in maintaining DNA replication and genome integrity. During mitosis, the mitotic checkpoint complex exerts strict surveillance on the fidelity of the mitotic process by sensing the proper tension between kinetochores of paired chromatids and the attachment of bipolar microtubules to kinetochores (3). BubR1, one of the mitotic checkpoint complex proteins, plays dual roles in sensing unaligned chromatids at the kinetochore and in suspending E3 ubiquitin ligase function in the cytoplasm $(4,5)$. In this way, the mitotic spindle checkpoint ensures that sister-chromatids can be evenly distributed into daughter cells to sustain their genetic integrity. Otherwise, BubR1 is activated and hyperphosphorylated to arrest the mitotic process by inhibiting metaphase-anaphase transition. Consequently, prolonged mitotic arrested cells exit mitosis and undergo postmitotic apoptosis. Abnormal expression of mitotic checkpoint proteins, such as Mad2 and Bub1, has been widely reported to be related to the chromosomal instability of cells and tumor predisposition $(6,7)$. Overexpression of BubR1 has been reported in several types of malignancies, including breast, bladder, gastric, ovarian, kidney and esophageal squamous cell cancers (8-13), while mutations of the Bublb gene are rarely observed (14). Depletion of BubR1 protein or loss of function 
can cause spindle checkpoint failure and further disturb the normal mitotic process, which finally results in chromosomal instability and aneuploidy $(11,15)$. Furthermore, haploinsufficiency of BubR1 in mice can trigger tumorigenesis and senescence $(16,17)$. Therefore, it is likely that abnormal expression of BubR1 may be involved in tumor progression and chemotherapeutic responsiveness.

Paclitaxel is a potent chemotherapeutic agent approved to treat ovarian, breast and lung cancers. As an adjuvant chemotherapy drug, paclitaxel has recently been used to potentiate the efficacy of 5-fluorouracil and cisplatin in esophageal cancer therapy. Several studies have shown that the cytotoxic effects of paclitaxel depend on the proper and timely activation of mitotic checkpoints (18-20). Defective mitotic spindle checkpoint by gradually reducing BubR1 in SKOV3-TR30 cells was found to lead to paclitaxel resistance through decreasing the mitotic index and suppressing cell death (21). However, the depletion or inactivation of BubR1 in several cancer cell lines somehow leads to an increase in their sensitivity to paclitaxel (22). Thus, it remains unclear how the expression status of BubR1 in cancer cells would affect the development of paclitaxel resistance. Nonetheless, it was reported that esophageal squamous cell cancer TE1 cells with relatively low BubR1 expression show more sensitivity to paclitaxel than TE2 cells (13).

This study was designed to thoroughly evaluate the role of mitotic checkpoint protein BubR1 in the development of paclitaxel chemoresistance in esophageal cancer cells. Using quantitative real-time PCR analysis on 50 samples of paired esophageal squamous cell cancer (ESC) tissues and adjacent non-cancerous tissues, we found that $72 \%$ (36 of 50) of the analyzed ESC samples exhibited a high expression level of BubR1, which was also confirmed in ESC cell lines. ESC cells with a high level of BubR1 expression were less sensitive to the anti-microtubule drugs paclitaxel and nocodazole. Recombinant adenovirus-mediated enforced expression of BubR1 in relatively sensitive ESC cell lines resulted in increased resistance to paclitaxel. Conversely, RNAi-mediated knockdown of BubR1 restored the ESC cell sensitivity to paclitaxel. Cell cycle analysis indicated that the sub-G1 cell population increased in the ESC cells having a reduced BubR1 level. Our results suggest that upregulation of BubR1 expression may be associated with ESC resistance to paclitaxel treatment. Thus, BubR1 may serve as a potential chemosensitizing target to overcome chemoresistance in ESC.

\section{Materials and methods}

Esophageal cancer samples. The use of clinical esophageal cancer samples was approved by the Institutional Ethics Committees of Chongqing Medical University (Chongqing, China) and Chuanbei Medical College (Sichuan, China). The patients consented to the use of the surgically resected samples for this study. Tissue specimens $(\mathrm{n}=50)$ from freshly resected esophageal squamous cell cancer and adjacent non-cancerous tissues were obtained at the Affiliated Hospital of Chuanbei Medical College. Samples were maintained in liquid nitrogen and used for RNA extraction using TRIzol agent (Invitrogen).

Cell culture and chemicals. ESC cell lines ECA-109, KYSE150 and KYSE180 were cultured in RPMI-1640 medium (Hyclone) supplemented with $10 \%$ fetal bovine serum (FBS, Hyclone), and the breast cancer cell line MCF-7 was cultured in DMEM (Hyclone) in $10 \%$ FBS. All cells were maintained in 1\% penicillin/streptomycin in $5 \% \mathrm{CO}_{2}$ at $37^{\circ} \mathrm{C}$. Paclitaxel and nocodazole were purchased from Sangon Biotech (Shanghai, China).

Recombinant adenovirus construction and amplifications. Recombinant adenovirus-expressing hBubR1 was generated as previously described (23). Briefly, the coding region for BubR1 was amplified by PCR and subcloned into a shuttle vector for recombinant adenovirus generation using the AdEasy system (24). Then, recombinant adenoviruses were packaged and amplified in HEK-293 cells. High titer of adenovirus expression of both GFP and BubR1 was obtained, namely AdBubR1, while adenovirus only expressing GFP (i.e., AdGFP) was used as a negative control.

$R N A$ interference. Oligo cassettes for short hairpin RNA (shRNA) targeting BubRI mRNA (NM_001211.4) were synthesized by GenePharma (Shanghai, China) and cloned into the pU6/GFP/Neo vector. Four pairs of shRNA oligos targeting 606-626, 1518-1538, 1745-1765 and 2564-2584 nucleotides of BubR1 mRNA were constructed. A scramble shRNA cassette was constructed as the negative control. The shRNA vectors were transfected into cells using Lipofectamine 2000 (Invitrogen) according to the manufacturer's instructions. The efficacy of RNA interference was verified by western blot analysis and RT-PCR assays of transfected cells.

$R N A$ extraction and quantitative real-time PCR. Total RNA of the clinical samples was isolated using TRIzol reagent. RNA quality and integrity were verified using the NanoDrop 1000 spectrophotometer (Thermo Scientific, Inc.) and RNA gel electrophoresis. Two micrograms of total RNA of each sample was reverse transcribed to cDNA with random hexamer primers and the Prime Script kit (Takara). Quantitative PCR was performed using Rotor Gene 6000 (Corbett Life Science, Qiagen). Each sample was analyzed in triplicate. PCR primers were as follows: BubR1, 5'-ACGTTATTAGAAAGAGCTGTAG-3' (forward) and 5'-CATATCCAAAGGCTCATTGC-3' (reverse); GAPDH, 5'-CAGCGACACCCACTCCTC-3' (forward) and 5'-TGAGGTCCACCACCCTGT-5' (reverse). Gene expression was quantified using SYBR-Green I dye real-time detection system. Thermal cycling conditions consisted of an initial $2 \mathrm{~min}$ at $95^{\circ} \mathrm{C}$, followed by 40 cycles for $20 \mathrm{sec}$ at $95^{\circ} \mathrm{C}$ and $20 \mathrm{sec}$ at $57^{\circ} \mathrm{C}$. Quantification was based on the relative expression ratio of BubR1 in esophageal cancer tissue to adjacent cancerous tissue after normalizing with internal GAPDH expression.

Western blot analysis. Cells were lysed with RIPA lysis buffer (BiYunTian, Shanghai, China) plus $1 \mathrm{mmol}$ PMSF on ice for $30 \mathrm{~min}$, and centrifuged at $13000 \mathrm{x} \mathrm{g}$ at $4^{\circ} \mathrm{C}$ for an additional $30 \mathrm{~min}$. Protein concentrations were determined using the NanoDrop 1000 spectrophotometer (Thermo Scientific, Inc.). Each sample containing $30 \mu \mathrm{g}$ total proteins was resolved by $8 \%$ SDS-PAGE and transferred onto PVDF membranes (Millipore). Primary BubR1 antibody (1:2000, mouse monoclonal antibody, BD Biosciences) and anti- $\beta$ tubulin (1:1000, Santa Cruz Biotechnology) were diluted in TBST buffer, 
respectively, and applied on the membranes for overnight incubation at $4^{\circ} \mathrm{C}$. The PVDF membranes were incubated with horseradish peroxidase-conjugated secondary antibody (goat; ZhongShan Goldenbridge Biotechnology, China) for $1 \mathrm{~h}$ at room temperature. After extensive washes with TBST, the protein bands of interest were visualized using space chemoluminescence reagent (Pierce) in the ChemiDoc ${ }^{\mathrm{TM}} \mathrm{XRS}+$ system (Bio-Rad, USA).

Cell viability assay. Cytotoxicity was determined by MTT assays (Promega). Briefly, 5000 cells/well were seeded into 96-well plates in $100 \mu \mathrm{l}$ culture medium. The medium was then replaced by various concentrations of paclitaxel. At 24, 48 and $72 \mathrm{~h}$ after treatment, $20 \mu \mathrm{l}$ of $5 \mathrm{mg} / \mathrm{ml}$ MTT reagent was added to each well and incubated for $4 \mathrm{~h}$ at $37^{\circ} \mathrm{C}$. MTT formazan precipitates were completely dissolved in DMSO with agitation. Optical density of each sample was measured at $490 \mathrm{~nm}$ with a plate reader (Sunrise Remote; Tecan, Switzerland).

Mitotic index analysis. ESC cells were treated with $0.2 \mu \mathrm{g} / \mathrm{ml}$ nocodazole for the indicated time points and harvested via trypsinization. Cells were suspended in a hypotonic solution containing $0.075 \mathrm{M} \mathrm{KCL}$ in PBS to swell for $15 \mathrm{~min}$ at $37^{\circ} \mathrm{C}$. After centrifugation, pellets were fixed in three changes of ice cold newly prepared methanol/acetic acid (3:1), and then incubated in fixative at $4^{\circ} \mathrm{C}$ overnight. Metaphase spreads were prepared by dropping cell pellets onto glass slides and staining with Giemsa and after the slides were air dried at $37^{\circ} \mathrm{C}$. At least 500 cells were counted in triplicate for each sample. Mitotic and interphase cells were scored, and the mitotic index was determined by dividing the number of mitotic cells by the number of total counted cells.

Cell cycle analysis with FACS. Approximately $1 \times 10^{5}$ cells were plated in $6-\mathrm{cm}$ dishes for $24 \mathrm{~h}$. Paclitaxel was added (final concentration of $0.1 \mu \mathrm{M}$ ). Both floating and adherent cells were harvested at the indicated time points by trypsinization, washed 3 times with PBS and then fixed with 70\% ethanol/PBS while gently vortexing the tube. Cells were fixed at $4^{\circ} \mathrm{C}$ overnight and subjected to flow cytometric analysis.

Statistical analysis. The clinical features of the groups with different BubR1 expression were analyzed by the $\chi^{2}$ test $(2 \times 2$ or $\mathrm{RxC}$ contingency tables). Analysis of BubR1 relative expression was performed using real-time quantitative PCR and $2^{-\triangle \Delta C T}$ method. Other comparisons were carried out using the independent sample t-test. A P-value $<0.05$ was considered to indicate a statistically significant difference. All data were analyzed using SPSS 19.0.

\section{Results}

High expression of BubRl in clinical esophageal cancer samples. We collected 50 cases of clinical ESC samples and analyzed the relative expression of BubR1 between the cancer tissues and adjacent non-cancerous tissues by quantitative RT-PCR. As shown in Fig. 1, BubR1 was significantly overexpressed in the ESC cancer compared to the adjacent non-cancerous tissues. Seventy-two percent (36 of 50) of the samples showed relatively high BubR1 transcription $(\mathrm{P}<0.05)$,

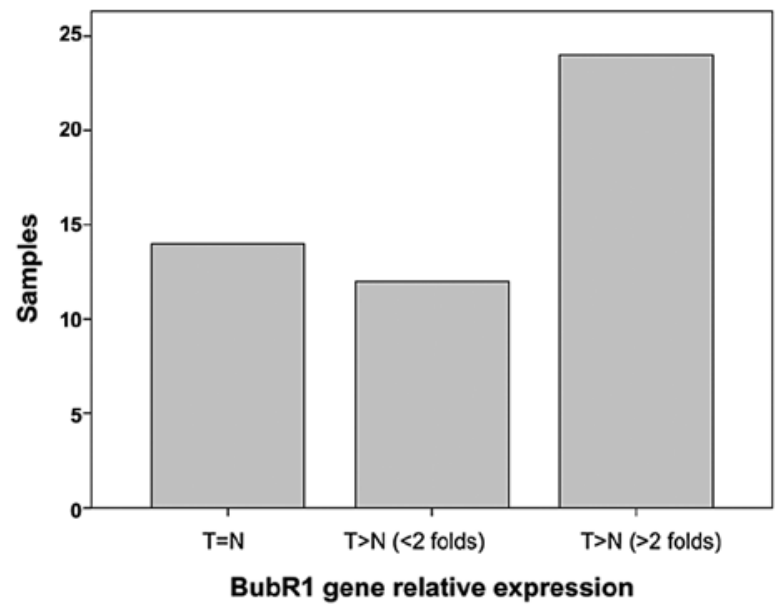

Figure 1. Expression of BubR1 in tumor (T) vs. normal-paired (N) samples Fifty cases of esophageal squamous cell cancer samples and their adjacent non-cancerous tissues were assessed. Total RNA was isolated and analyzed by real-time quantitative RT-PCR to detect the difference in BubR1 expression.

Table I. Patient characteristics and BubR1 expression.

\begin{tabular}{ccc}
\hline & \multicolumn{2}{c}{ BubR1 expression $(\mathrm{n}=50)$} \\
\cline { 2 - 3 } Features & $\begin{array}{c}\text { Unchanged } \\
(\mathrm{n}=14)\end{array} \quad \begin{array}{c}\text { Increased } \\
(\mathrm{n}=36)\end{array}$ & P-value
\end{tabular}

Age (years), mean $\pm \mathrm{SD}$

$60.3 \pm 7.9$

Gender

Male

12

27

Female

2

9

Histopathologic grading

Well differentiation

18

Moderate differentiation

8

12

Poor differentiation

5

Lymphatic invasion

1

Negative

8

22

Positive

6

14

Stage

I

II

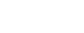

III

$5 \quad 16$

IV

2

0.196

Location of tumor

Upper

Middle

0

1

Lower

9

30

$5 \quad 5$

and 64\% (23 of 36) of those samples exhibited at least a 2-fold higher BubR1 level than that the in non-cancerous tissues.

Previous studies have demonstrated that upregulation of BubR1 in ovarian and tonsillar cancers was associated with lymph node metastasis, tumor grade and stage $(11,25)$. We examined the relationship between the clinical histopathological features and BubR1 expression in the ESC cases (Table I). 
A

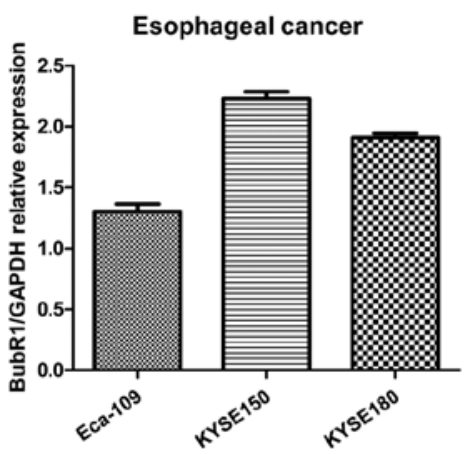

B
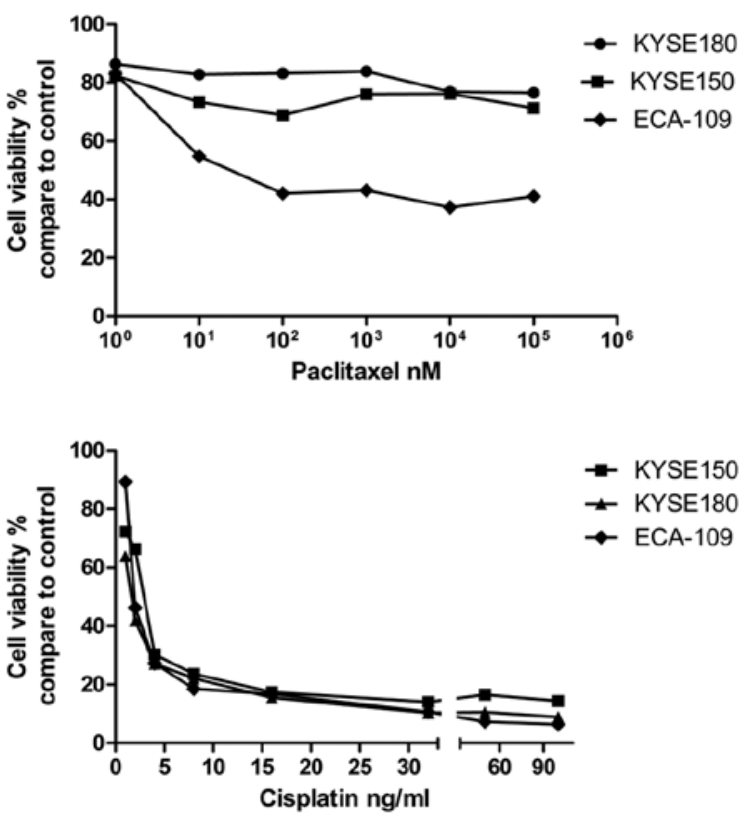

C
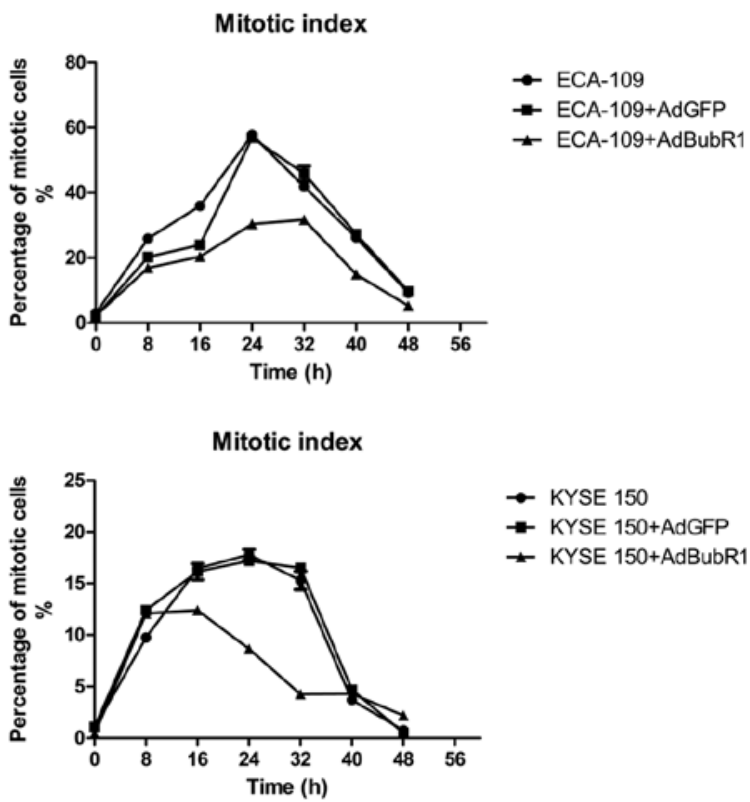

$\rightarrow$ KYSE 150

$\rightarrow$ KYSE 150+AdGFP

- KYSE 150+AdBubR

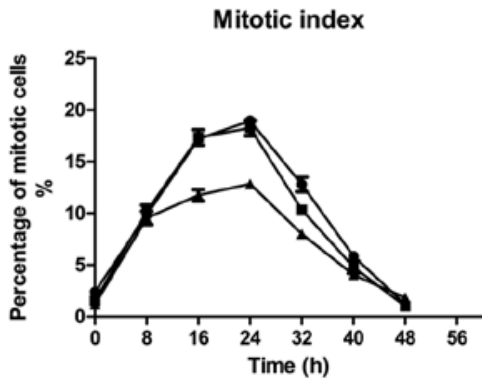

$\rightarrow$ KYSE180

$\rightarrow$ KYSE180+AdGFP

- KYSE180+AdBubR

Figure 2. High BubR1 expression in esophageal squamous cell cancer cell lines is associated with relative resistance to paclitaxel and impaired mitotic progression. (A) BubR1 transcription levels in esophageal cancer cell lines ECA-109, KYSE150 and KYSE180 were determined by real-time quantitative RT-PCR. Clearly, KYSE150 and KYSE180 showed relatively high BubR1 expression compared with ECA-109. (B) Under various concentrations of paclitaxel, the cell viability was determined by MTT assay. (C) Mitotic index assay was carried out using $0.2 \mu \mathrm{g}$ nocodazole for the indicated time points.

No significant correlations were found between BubR1 expression levels and the clinical histopathological parameters.

Correlation of the high expression of BubR1 in ESC cell lines with their resistance to anti-microtubule drugs. Mitotic checkpoint protein BubR1 has been reportedly involved in the cytotoxic effects of anti-microtubule drugs. We tested whether high BubR1 expression in ESC cells correlates with their paclitaxel sensitivity. We first determined the BubR1 mRNA levels in three ESC cell lines using quantitative RT-PCR (Fig. 2A). Consistent with the results from the clinical samples, all three cell lines exhibited a high level of BubR1 expression. Compared with ECA-109, the KYSE150 and KYSE180 cell lines exhibited relatively higher expression of BubR1. Drug sensitivity assay demonstrated that KYSE150 and KYSE180 cells were less responsive to paclitaxel than ECA-109 (Fig. 2B). Cisplatin is clinically used for esophageal cancer treatment and served as a positive control. The responsiveness of cancer cells to paclitaxel is potentially linked to mitotic progression and the functional mitotic checkpoint. The mitotic index analysis showed that much fewer mitotic cells were noted in the
KYSE150 and KYSE180 cell lines when treated with the antimicrotubule agent nocodazole, suggesting that the paclitaxel insensitivity and mitosis progression may be linked in these ESC cell lines. Accordingly, when BubR1 was overexpressed via an adenoviral vector AdBubR1, all three cell lines showed a severe disrupted mitotic progression (Fig. 2C). Thus, high BubR1 expression may be closely associated with paclitaxel resistance.

Restoration of paclitaxel sensitivity by RNAi-mediated BubRl knockdown in esophageal cancer cells. To further explore the possibility that a high BubR1 level may lead to paclitaxel insensitivity, we constructed expression vectors that contained four different shRNA pairs targeting the BubR1 gene and a control shRNA with a scramble sequence, respectively. These shRNA vectors were transfected into ECA-109 cells and the interference effects were determined by quantitative PCR and western blot assays. As shown in Fig. 3A, the BubR1 transcription level was significantly reduced in the si-BubR1-4 lane, which was also confirmed by western blot analysis. The three ESC lines were transfected 
A
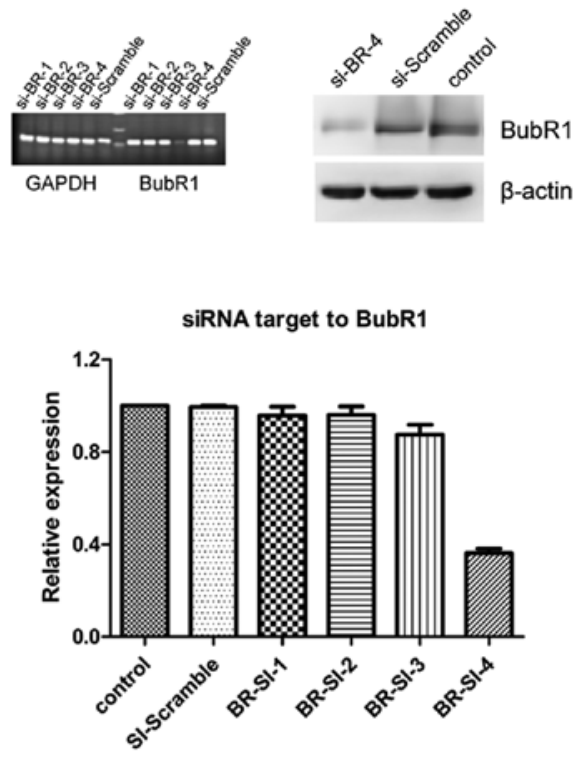

C

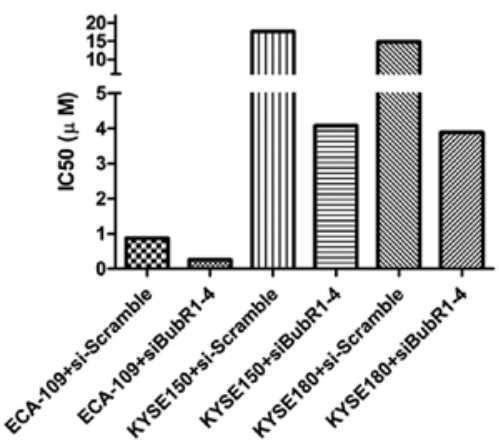

B

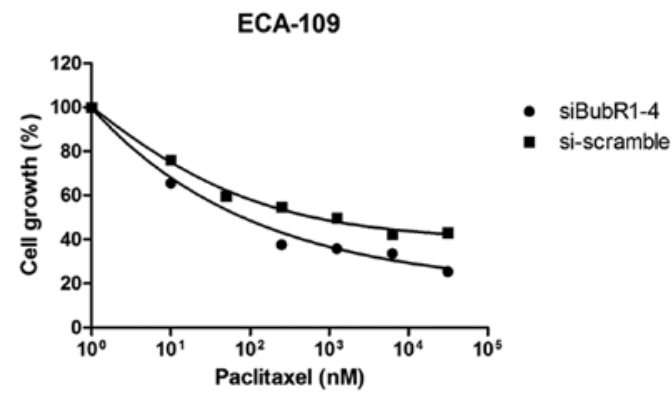

KYSE 150

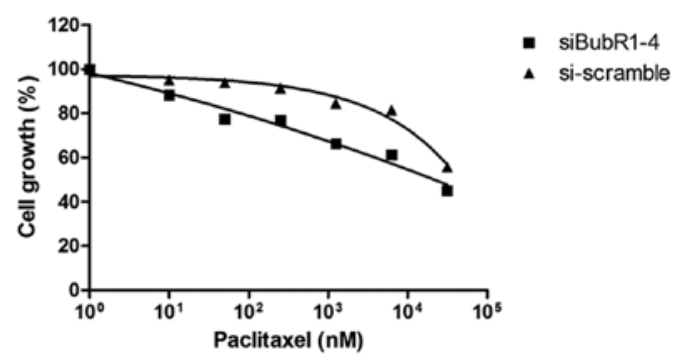

KYSE 180

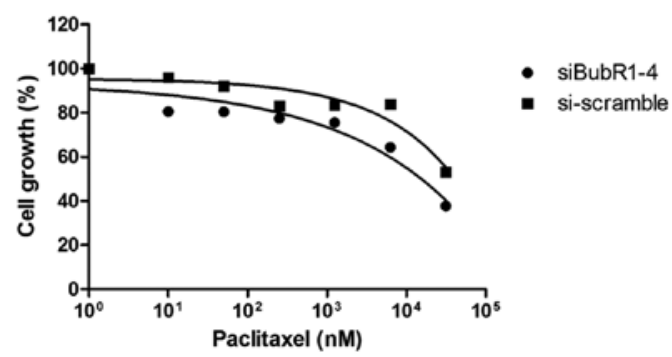

Figure 3. Knockdown of BubR1 moderately restores the sensitivity of esophageal squamous cancer cells to paclitaxel. (A) Results from real-time quantitative or regular RT-PCR and western blot assays indicated that plasmids pU6/GFP/Neo-shRNA-BubR1-4 contained an effectively interfering sequence to BubR1. (B) Three esophageal cancer cell lines were transfected with the si-BubR1-4 plasmid or si-Scramble as a negative control for $24 \mathrm{~h}$ and treated with various concentrations of paclitaxel for $48 \mathrm{~h}$. Cell viability was assessed. (C) $\mathrm{IC}_{50}$ of every group was calculated, and reduced $\mathrm{IC}_{50}$ values were observed in cells transfected with the si-BubR1-4 plasmid.

with si-BubR1-4 or si-Scramble plasmids. At $24 \mathrm{~h}$, the transfected cells were treated with paclitaxel. BubR1 suppression in the three cell lines led to a moderate restoration of drug sensitivity, and the respective drug $\mathrm{IC}_{50}$ values were reduced (Fig. 3C), (i.e., ECA-109, from 0.86 to $0.26 \mu \mathrm{M}$; KYSE150, from 17.68 to $4.07 \mu \mathrm{M}$; KYSE 180 , from 14.82 to $3.88 \mu \mathrm{M}$; $\mathrm{P}<0.05)$. We next sought to determine whether increased drug sensitivity was associated with cell death. ECA-109 cells transfected with siRNA were treated with $100 \mathrm{nM}$ paclitaxel for 24 or $48 \mathrm{~h}$, respectively, followed by the FACS assay. FACS results showed that cell death was more severe in the si-BubR1-4 group (Fig. 4).

Induction of drug resistance by forced expression of BubR1. As most ESC cell lines have an elevated BubR1 level and are insensitive to anti-microtubule agents, we further tested whether forced expression of BubR1 would confer drug resistance in the cancer cells that were relatively sensitive to paclitaxel. We transduced the breast cancer cell line MCF-7 with AdBubR1. The infected cells were subjected to cell proliferation and drug responsiveness assays. As shown in Fig. 5, overexpression of BubR1 did not affect MCF-7 cell proliferation, but significantly increased the paclitaxel resistance of the infected MCF-7 cells.

\section{Discussion}

Spindle assembly checkpoint (SAC), orchestrated by multiple proteins, is a highly conversed surveillance mechanism that exists in mitosis. BubR1 is one of the most important mitotic phosphoproteins which serves both as a sensor to coordinate with CENP-E for proper attachment between microtubules and the kinetochore, and as an executor to delay the onset of the anaphase by halting APC/C activity. Several clinical studies have indicated that abnormal BubR1 expression is prevalent in cancers and is somehow associated with tumor progression and prognosis (8-13). BubR1 upregulation in esophageal cancer has also been reported and cells with high BubR1 expression 
A

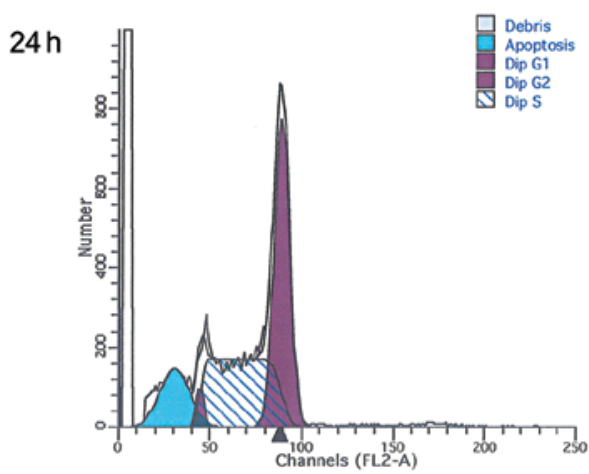

$48 \mathrm{~h}$

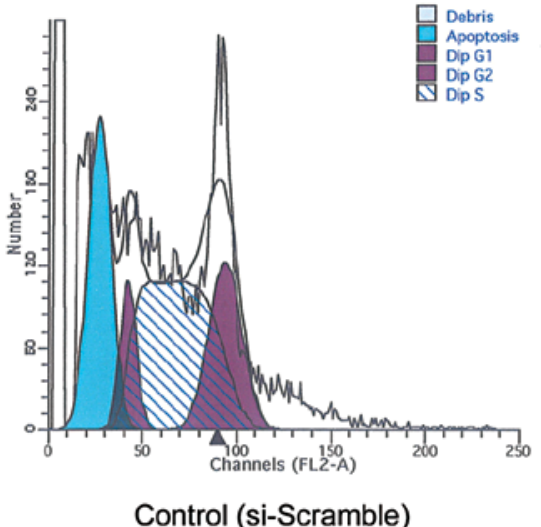

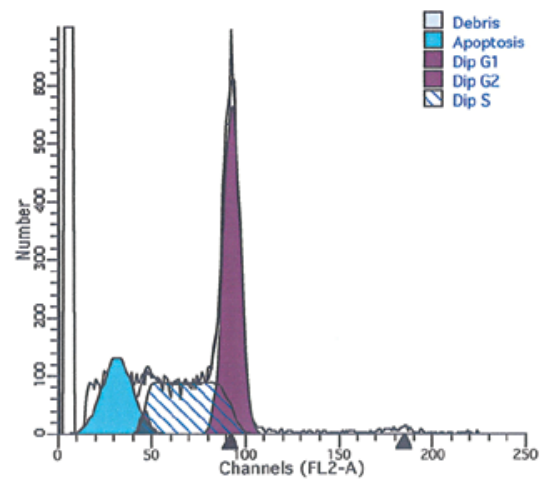

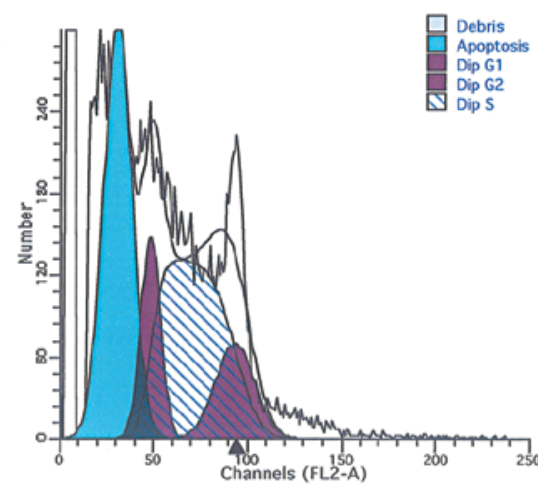

Expreiment (si-BubR1-4)
B

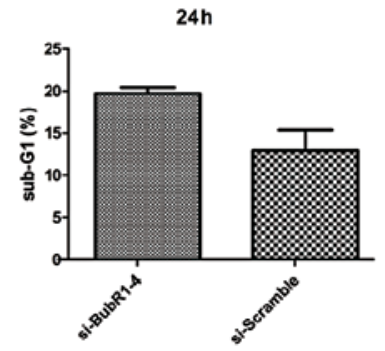

$48 \mathrm{~h}$

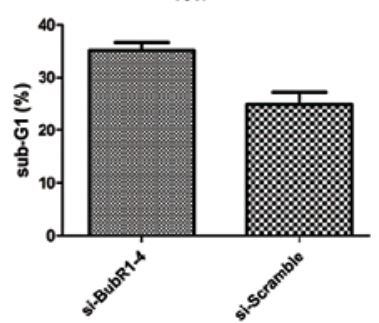

Figure 4. Suppression of BubR1 in esophageal squamous cell cancer cells induces increased cell death following paclitaxel exposure. (A) Cell cycle analysis was determined in ECA-109 cells transfected with the si-BubR1-4 plasmid and treated with $0.1 \mu \mathrm{M}$ paclitaxel for 24 and 48 h, respectively. (B) Percentages of cells in the sub-G1 phase are shown. At 24 and $48 \mathrm{~h}$, the distribution of cells in the sub-G1 phase in the control group (si-Scramble) was 10.28 and $27.26 \%$, and the distribution of cells in the sub-G1 phase in the experiment group (si-BubR1-4) was 15.34 and $37.67 \%$, respectively $(\mathrm{P}<0.05)$.

A

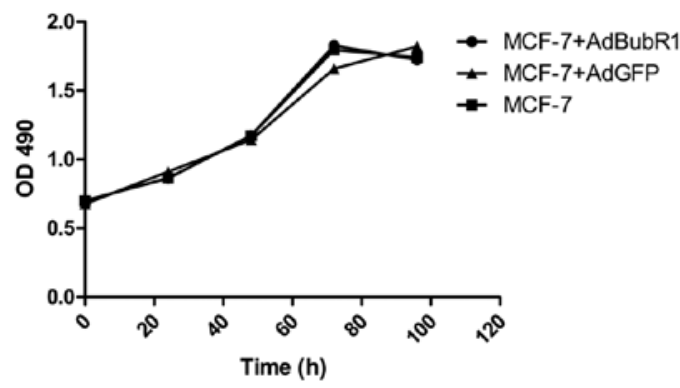

B

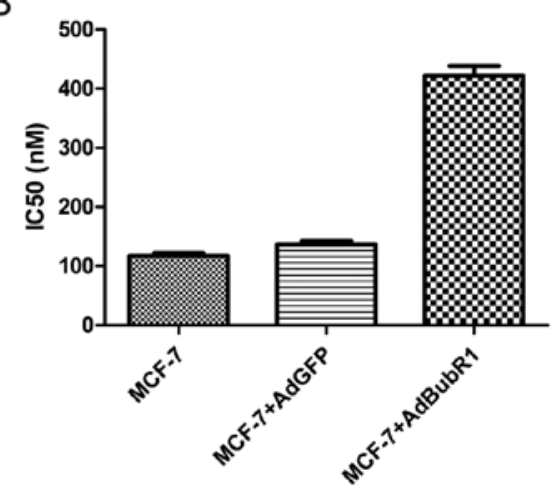

Figure 5. Forced BubR1 expression leads to resistance to paclitaxel. (A) Overexpression of BubR1 by infection of the breast cancer cell line MCF-7 with AdBubR1 or AdGFP had no influence on the cell proliferative ability. (B) Upregulation of BubR1 in MCF-7 cells caused paclitaxel resistance.

exhibit relatively low sensitivity to paclitaxel. Here, we found that BubR1 expression was greatly upregulated in clinical esophageal squamous cell cancer samples compared with respective adjacent non-cancerous tissues, although statistical analysis failed to identify any correlations between BubR1 expression and tumor progression, or lymphatic invasion. It is conceivable that BubR1 is not sufficient to serve as an independent prognostic factor, but may be potentially valuable in combination with other factors, as shown in the cases of breast and colon cancer $(8,26)$. While $h B U B l b$ mutations are rarely detected in clinical cancers, it remains to be determined what causes BubR1 overexpression in a variety of cancers. However, several findings may shed light on this issue. At the early stage of esophageal cancer development, aberrant expression of cell cycle regulators has previously been found in addition to oncogene mutations. Analysis of the $h B U B 1 b$ promoter region in lung cancer cell lines found multiple transcription factor binding sites (27). p53 and Myc proteins have been found to 
interact with the $h B U B 1 b$ promoter and regulate its transcription (28). In addition, the status of $h B U B l b$ promoter methylation in tumors may indirectly regulate BubR1 expression (29). Thus, BubR1 overexpression is likely the consequence of an interaction of many regulatory signaling complexes.

High BubR1 expression in gastric cancer was significantly correlated with DNA aneuploidy (10). We found that forced BubR1 overexpression in esophageal cancer cel lines resulted in disrupted mitotic progression. Consistent with a previous study (13), BubR1 overexpression is easily detected in esophageal squamous cell cancer samples. Moreover, esophageal squamous cell cancer cell lines with high BubR1 expression are seemingly insensitive to paclitaxel.

As a potent anti-microtubule drug, paclitaxel which requires a functional mitotic checkpoint is one of the most commonly used chemotherapeutic drugs in the clinic. However, its application and anticancer efficacy are limited by its rapidly developed drug resistance. The events that ensure that cells enter into mitosis and that the mitotic checkpoint is properly activated, no matter how long it lasts, guarantee anti-microtubule drug efficiency $(19,30)$. Therefore, paclitaxel sensitivity will be affected under the condition of mitotic checkpoint failure caused by abnormal checkpoint protein expression. Although BubR1 suppression severely impairs the mitotic checkpoint and confers drug resistance to cancer cells, forced expression of BubR1 also leads to mitotic checkpoint dysfunction and reduces drug sensitivity, as shown in our studies.

Conversely, an RNAi-mediated depletion of BubR1 in esophageal cancer cells contributes to a modest restoration of paclitaxel sensitivity, as shown by a reduction in $\mathrm{IC}_{50}$ values and accumulation of the sub-G1 fraction. In cell lines derived from breast and ovarian cancers, which also exhibit high BubR1 expression in clinical samples, a decreased BubR1 level is associated with augmented paclitaxel-induced cell death $(20,21)$. Thus, it is conceivable that either suppression or promotion of BubR1 expression can disrupt the function of the mitotic checkpoint and may subsequently diminish the effects of paclitaxel. In the case of esophageal squamous cell cancer, overexpression of BubR1 is seemingly a dominant event, conferring cancer cells with insensitivity to paclitaxel. Thus, future studies should be directed to determine the role of BubR 1 expression in the development of drug resistance against anti-microtubule drugs.

In summary, our study revealed that mitotic checkpoint BubR1 is overexpressed in clinical esophageal squamous cell cancer tissues and in esophageal squamous cell cancer cell lines. Esophageal squamous cell cancer cells with high BubR1 expression show resistance to the anti-microtubule drug paclitaxel, while knockdown of BubR1 significantly sensitizes the responsiveness of these cells to paclitaxel. Thus, our findings strongly suggest that BubR1 may serve as a potential chemosensitizing target to overcome the chemoresistance of ESC.

\section{Acknowledgements}

The reported study was supported in part by research grants from the Special Academic Foundation of Doctor Degrees of Chongqing Medical University funded by the Ministry of Education of China (No. 20115503110009), and was supported by grants 30872770 from the National Natural Science Foundation of China (NSFC30872770), Natural Science Foundation Project of CQ CSTC (CSTC, 2011BB5131) and the National Ministry of Education Foundation of China (KJ120327).

\section{References}

1. Parkin DM: Global cancer statistics in the year 2000. Lancet Oncol 2: 533-543, 2001.

2. Stathopoulos GP and Tsiaras N: Epidemiology and pathogenesis of esophageal cancer: management and its controversial results (Review). Oncol Rep 10: 449-454, 2003.

3. Musacchio A and Salmon ED: The spindle-assembly checkpoint in space and time. Nat Rev Mol Cell Biol 8: 379-393, 2007.

4. Bolanos-Garcia VM and Blundell TL: BUB1 and BUBR1: multifaceted kinases of the cell cycle. Trends Biochem Sci 36: 141-150, 2011.

5. Elowe S, Dulla K, Uldschmid A, Li X, Dou Z and Nigg EA: Uncoupling of the spindle-checkpoint and chromosomecongression functions of BubR1. J Cell Sci 123: 84-94, 2010.

6. Sotillo R, Hernando E, Díaz-Rodríguez E, Teruya-Feldstein J, Cordón-Cardo C, Lowe SW and Benezra R: Mad2 overexpression promotes aneuploidy and tumorigenesis in mice. Cancer Cell 11: 9-23, 2007.

7. Ricke RM, Jeganathan KB and van Deursen JM: Bub1 overexpression induces aneuploidy and tumor formation through Aurora B kinase hyperactivation. J Cell Biol 193: 1049-1064, 2011.

8. Yuan B, Xu Y, Woo JH, et al: Increased expression of mitotic checkpoint genes in breast cancer cells with chromosomal instability. Clin Cancer Res 12: 405-410, 2006.

9. Yamamoto Y, Matsuyama H, Chochi Y, et al: Overexpression of BUBR1 is associated with chromosomal instability in bladder cancer. Cancer Genet Cytogen 174: 42-47, 2007.

10. Ando K, Kakeji Y, Kitao H, et al: High expression of BUBR1 is one of the factors for inducing DNA aneuploidy and progression in gastric cancer. Cancer Sci 101: 639-645, 2010.

11. Lee YK, Choi E, Kim MA, Park PG, Park NH and Lee H: BubR1 as a prognostic marker for recurrence-free survival rates in epithelial ovarian cancers. Br J Cancer 101: 504-510, 2009.

12. Pinto M, Vieira J, Ribeiro FR, et al: Overexpression of the mitotic checkpoint genes BUB1 and BUBR1 is associated with genomic complexity in clear cell kidney carcinomas. Cell Oncol 30: 389-395, 2008

13. Tanaka K, Mohri Y, Ohi M, et al: Mitotic checkpoint genes, hsMAD2 and BubR1, in oesophageal squamous cancer cells and their association with 5-fluorouracil and cisplatin-based radiochemotherapy. Clin Oncol (R Coll Radiol) 20: 639-646, 2008.

14. Hanks S, Coleman K, Reid S, et al: Constitutional aneuploidy and cancer predisposition caused by biallelic mutations in BUB1B. Nat Genet 36: 1159-1161, 2004.

15. Dai W, Wang Q, Liu T, et al: Slippage of mitotic arrest and enhanced tumor development in mice with BubR1 haploinsufficiency. Cancer Res 64: 440-445, 2004.

16. Baker DJ, Perez-Terzic C, Jin F, et al: Opposing roles for p16Ink4a and p19Arf in senescence and ageing caused by BubR1 insufficiency. Nat Cell Biol 10: 825-836, 2008.

17. Baker DJ, Jeganathan KB, Cameron JD, et al: BubR1 insufficiency causes early onset of aging-associated phenotypes and infertility in mice. Nat Genet 36: 744-749, 2004.

18. Kim M, Murphy K, Liu F, Parker SE, Dowling ML, Baff W and Kao GD: Caspase-mediated specific cleavage of BubR1 is a determinant of mitotic progression. Mol Cell Biol 25: 9232-9248, 2005.

19. Sudo T, Nitta M, Saya H and Ueno NT: Dependence of paclitaxel sensitivity on a functional spindle assembly checkpoint. Cancer Res 64: 2502-2508, 2004

20. Lee EA, Keutmann MK, Dowling ML, Harris E, Chan G and Kao GD: Inactivation of the mitotic checkpoint as a determinant of the efficacy of microtubule-targeted drugs in killing human cancer cells. Mol Cancer Ther 3: 661-669, 2004.

21. Fu Y, Ye D, Chen H, Lu W, Ye F and Xie X: Weakened spindle checkpoint with reduced BubR1 expression in paclitaxel-resistant ovarian carcinoma cell line SKOV3-TR30. Gynecol Oncol 105: 66-73, 2007. 
22. Yamada HY and Gorbsky GJ: Inhibition of TRIP1/S8/hSug1, a component of the human $19 \mathrm{~S}$ proteasome, enhances mitotic apoptosis induced by spindle poisons. Mol Cancer Ther 5: 29-38, 2006.

23. Shin HJ, Baek KH, Jeon AH, et al: Dual roles of human BubR1, a mitotic checkpoint kinase, in the monitoring of chromosomal instability. Cancer Cell 4: 483-497, 2003.

24. Luo J, Deng Z-L, Luo X, et al: A protocol for rapid generation of recombinant adenoviruses using the AdEasy system. Nat Protoc 2: 1236-1247, 2007.

25. Hannisdal K, Burum-Auensen E, Schjolberg A, De Angelis PM and Clausen OP: Correlation between reduced expression of the spindle checkpoint protein BubR1 and bad prognosis in tonsillar carcinomas. Head Neck 32: 1354-1362, 2010.

26. Rao CV, Yang YM, Swamy MV, et al: Colonic tumorigenesis in $\mathrm{BubR}^{+/-} \mathrm{Apc}^{\mathrm{Min} /+}$ compound mutant mice is linked to premature separation of sister chromatids and enhanced genomic instability. Proc Natl Acad Sci USA 102: 4365-4370, 2005.
27. Seike M, Gemma A, Hosoya Y, et al: The promoter region of the human BUBR1 gene and its expression analysis in lung cancer. Lung Cancer 38: 229-234, 2002.

28. Menssen A, Epanchintsev A, Lodygin D, et al: c-MYC delays prometaphase by direct transactivation of MAD2 and BubR1: identification of mechanisms underlying c-MYC-induced DNA damage and chromosomal instability. Cell Cycle 6: 339-352, 2007.

29. Park HY, Jeon YK, Shin HJ, et al: Differential promoter methylation may be a key molecular mechanism in regulating BubR1 expression in cancer cells. Exp Mol Med 39: 195-204, 2007.

30. Wu YC, Yen WY and Yih LH: Requirement of a functional spindle checkpoint for arsenite-induced apoptosis. J Cell Biochem 105: 678-687, 2008. 